\title{
Reduced Mass-Weighted Proper Decomposition for Modal Analysis
}

\author{
Venkata K. Yadalam \\ Department of Mechanical Engineering \\ Michigan State University \\ East Lansing, Michigan 48824 \\ Email: kalyan.612@gmail.com \\ B. F. Feeny \\ Department of Mechanical Engineering \\ Michigan State University \\ East Lansing, Michigan 48824 \\ Email: feeny@egr.msu.edu
}

\begin{abstract}
A method of modal analysis by a mass-weighted proper orthogonal decomposition for multi-degree-of-freedom and distributed-parameter systems of arbitrary mass distribution is outlined. The method involves reduced-order modeling of the system mass distribution so that the discretized mass matrix dimension matches the number of sensed quantities, and hence the dimension of the response ensemble and correlation matrix. In this case, the linear interpolation of unsensed displacements is used to reduce the size of the mass matrix. The idea is applied to the modal identification of a mass-spring system and an exponential rod.
\end{abstract}

\section{Introduction}

There are different ways to determine vibration mode shapes. Traditional modal analysis is performed by using frequency responses from input-output data [1,2]. Some time domain output-only methods are the Ibrahim time domain method [3], polyreference method [4], eigensystem realization algorithm [5], least square complex exponential method [6], and stochastic subspace identification methods [7]. Frequency based output-only methods include the orthogonal polynomial methods [8,9], complex mode indicator function [10], and frequency domain decomposition [11]. Some of these methods have been compared recently $[12,13]$. Proper orthogonal decomposition (POD) can be used to estimate linear normal modes under certain conditions [14-17], with success in experiments [18-20]. Recent variations of POD, the smooth orthogonal decomposition (SOD) [21] and state-variable modal decomposition [22] methods, have been developed for free response cases. POD is very simple and tends to be rather robust, but has room for development. In any case, it is best to apply a few of these tools

Our interest is in POD. Here POD for modal analysis is explained briefly for a simple linear problem. Suppose sensors detect the displacements of $n$ locations on the structure at $N$ different times and are stored in form of an $n \times N$ ensemble matrix, $\mathbf{X}$, whose rows represent each sampled displacement time history. The means are best removed from these signals in case unwanted bias exists. In POD, the $n \times n$ correlation matrix $\mathbf{R}=\frac{1}{N} \mathbf{X} \mathbf{X}^{T}$ is built, and the eigenvalue problem $\mathbf{R u}=\lambda \mathbf{u}$ is solved, yielding the POMs as the eigenvectors $\mathbf{u}$, and proper orthogonal values (POVs) as the eigenvalues $\lambda$. If the mass distribution is homogeneous, then the POMs represent the linear normal modes (LNMs).

If the mass matrix $\mathbf{M}$ is non-homogeneous, the mass-weighted proper decomposition

$$
\mathbf{R M u}=\lambda \mathbf{u}
$$

provides estimates of the LNMs from lightly damped, multimodal free responses. A stiffness-weighted decomposition can also be done, if the stiffness matrix $\mathbf{K}$ is known. In the mass-weighted case, the eigenvalues correspond to projections of 
mass-weighted data onto the POMs. To be more precise, consider for symmetric systems that the eigenvectors are orthogonal with respect to $\mathbf{M}$. Thus, from equation (1), $\mathbf{u}^{T} \mathbf{M}^{T} \mathbf{R} \mathbf{M u}=\lambda \mathbf{u}^{T} \mathbf{M}^{T} \mathbf{u}$. If the modal vectors are normalized with respect to $\mathbf{M}$, this equation can be rewritten as $\mathbf{u}^{T} \mathbf{M X X} \mathbf{X}^{T} \mathbf{M u} / N=\lambda$, or $\left(\mathbf{u}^{T} \mathbf{M X}\right)\left(\mathbf{u}^{T} \mathbf{M X}\right)^{T} / N=\lambda$. Noting that $\mathbf{X}=\left[\mathbf{x}\left(t_{1}\right), \mathbf{x}\left(t_{2}\right), \ldots, \mathbf{x}\left(t_{N}\right)\right]$, where $\mathbf{x}\left(t_{i}\right)$ is a displacement vector sampled at time $t_{i}$, then $\mathbf{u}^{T} \mathbf{M X}=\left[\mathbf{u}^{T} \mathbf{M} \mathbf{x}\left(t_{1}\right), \mathbf{u}^{T} \mathbf{M x}\left(t_{2}\right), \ldots, \mathbf{u}^{T} \mathbf{M x}\left(t_{N}\right)\right]$, and finally

$$
\frac{1}{N}\left(\mathbf{u}^{T} \mathbf{M X}\right)\left(\mathbf{u}^{T} \mathbf{M X}\right)^{T}=\frac{1}{N} \sum_{i=1}^{N}\left(\mathbf{u}^{T} \mathbf{M x}\left(t_{i}\right)\right)^{2}=\lambda,
$$

giving $\lambda$ the interpretation of mean squared projections of mass-weighted displacements onto the associated eigenvector, or alternatively, the mean squared projection of the data onto the vector Mu. With this information, in contrast to frequency information, the POD is well suited for studying modal dominance. Frequency information can be obtained by aforementioned related methods [21,22], constructing a related Rayleigh quotient [23], or looking at modal coordinates [18].

While eigenvalue problem (1) is not symmetric, the physics and mathematics imply that the eigensolution will be real. If $\mathbf{M}$ is positive definite and symmetric, then $\mathbf{M}^{1 / 2}$ and $\mathbf{M}^{-1 / 2}$ exist and are real. Letting $\mathbf{u}=\mathbf{M}^{-1 / 2} \underline{\psi}$, inserting into equation (1), and premultiplying by $\mathbf{M}^{1 / 2}$, leads to $\mathbf{M}^{1 / 2} \mathbf{R} \mathbf{M}^{1 / 2} \psi=\lambda \psi$, a real, symmetric eigenvalue problem with a real eigenvalues $\lambda$ and eigenvectors $\psi$. Hence the eigensolution for equation (1) is real.

Mass distributions may be known when the structures have simple geometry, or when the structural design or finite element analysis occurs in parallel with modal testing. A typical challenge might be that the mass distribution of the model (continuous or finite) may yield a mass matrix whose dimensions differ from the number of sensors, and hence the dimensions of $\mathbf{R}$. In this case, a reduced-order mass matrix needs to be approximated.

In this paper, we investigate the feasibility of a lumped-mass reduction of the mass matrix $\mathbf{M}$, such that the reduced mass matrix $\mathbf{M}_{r}$ is dimensionally compatible with the correlation matrix $\mathbf{R}$. We test the idea on one-dimensional examples, which are simple but well understood for interpretation.

\section{Formulation for Multi-Degree-of-Freedom Systems}

In this section, the fundamental foundation of the decomposition idea will be laid. The details of how the analysis is performed will then be done through an example, using displacement interpolation to achieve the mass matrix reduction.

\subsection{Foundation}

The equation of free vibration for a second order system is

$$
\mathbf{M} \ddot{\mathbf{x}}+\mathbf{K x}=\mathbf{0} .
$$

Suppose only some of the displacements, $\mathbf{y}$, are measured. We seek a reduced mass matrix corresponding to the measured displacements $\mathbf{y}$. First a matrix $\mathbf{W}$ of basis vectors is selected to represent an interpolation of the displacements $\mathbf{x}$ such that $\mathbf{x}=\mathbf{W y}$. Inserting into equation (3), and pre-multiplying by $\mathbf{W}^{T}$ we have

$$
\mathbf{W}^{T} \mathbf{M W} \ddot{\mathbf{y}}+\mathbf{W}^{T} \mathbf{K W y}=\mathbf{0} .
$$

From equation (4), the reduced-order mass matrix is $\mathbf{M}_{r}=\mathbf{W}^{T} \mathbf{M W}$ and the reduced stiffness matrix is $\mathbf{K}_{r}=\mathbf{W}^{T} \mathbf{K W}$. This process is applicable for any interpolating scheme for which the interpolated (unmeasured) displacements are linear combinations of the measured displacements. Examples of such schemes are Lagrange interpolation and cubic splines [24], and assumed-mode approximations [2,25]. The examples here will use simple linear (tent-function) interpolation to demonstrate the concept. Higher-order local interpolation has the capacity to improve results, for example as used spatially in finite element analysis, and in the time domain for limit cycle dynamics [26].

When the measured displacements $\mathbf{y}$ are sampled, the ensemble matrix $\mathbf{Y}$ is formed. We calculate the correlation matrix $\mathbf{R}=\frac{1}{N} \mathbf{Y} \mathbf{Y}^{T}$. We then perform the mass-weighted proper decomposition on $\mathbf{R M}_{r}$, via the unsymmetric eigenvalue problem

$$
\mathbf{R M}_{r} \mathbf{v}=\lambda \mathbf{v}
$$

The eigenvectors $\mathbf{v}$ are to estimate the structural modal vectors.

The following example illustrates a choice of $\mathbf{W}$ and a method for implementing it to find the reduced mass matrix $\mathbf{M}_{r}$. 


\subsection{Example}

We simulated an undamped system of masses $m_{1}=1 \mathrm{~kg}, m_{2}=1.25 \mathrm{~kg}, m_{2}=1.5 \mathrm{~kg}, \ldots, m_{24}=6.75 \mathrm{~kg}$, with displacements $x_{1}, x_{2}, x_{3}, \ldots, x_{24}$, connected by uniform springs of stiffness $k=1 \mathrm{~N} / \mathrm{m}$ in series, and grounded at both ends. With these parameters, the 24 undamped modal frequencies ranged from $0.0629 \mathrm{rad} / \mathrm{sec}$ to $1.7017 \mathrm{rad} / \mathrm{sec}$.

The displacements $\mathbf{y}=\left[x_{3}, x_{6}, x_{9}, \ldots, x_{24}\right]^{T}$ were taken to be measured. In this example, we chose the $24 \times 8$ basis matrix

$$
\mathbf{W}=\left[\begin{array}{ccccccccccc}
1 / 3 & 2 / 3 & 1 & 2 / 3 & 1 / 3 & 0 & 0 & 0 & 0 & \cdots & 0 \\
0 & 0 & 0 & 1 / 3 & 2 / 3 & 1 & 2 / 3 & 1 / 3 & 0 & \cdots & 0 \\
\vdots & & & & & \ddots & & & & \vdots \\
0 & \cdots & \cdots & 0 & 1 / 3 & 2 / 3 & 1 & 2 / 3 & 1 / 3 & 0 \\
0 & \cdots & & & & & 0 & 1 / 3 & 2 / 3 & 1
\end{array}\right]^{T}
$$

to linearly interpolate the unmeasured displacements between the sensed displacements $\mathbf{y}$. For example, interpolating the displacements between $x_{6}=y_{2}$ and $x_{9}=y_{3}$ produces $x_{7} \approx y_{2}+\left(y_{3}-y_{2}\right) / 3=2 y_{2} / 3+y_{3} / 3$ such that $W_{72}=2 / 3$ and $W_{73}=$ $1 / 3$.

We are interested in approximating the mode shapes of this system given the mass (alternatively the stiffness) matrix, and making use of the basis matrix and the measured free-vibration response. The eight sensors on the structure were sampled at a constant rate at $N=16000$ different times and were stored in an $8 \times N$ ensemble matrix $\mathbf{Y}$. The sampling rate was 0.1 s. As such, 16 periods of the fundamental mode were simulated, and about 37 samples per period of the highest-frequency mode were recorded.

For comparison of the POMs with the structural modes, the structural eigenvalue problem $\left(\mathbf{M}-\omega^{2} \mathbf{K}\right) \underline{\phi}=\mathbf{0}$, which, based on the full stiffness and mass matrices, gives us the natural modal matrix, $\Phi$, and eigenvalues, $\omega^{2}$.

Initial conditions determine which modes are dominant. Zero initial displacements, and initial velocities $\dot{\mathbf{x}}(0)=\left[\begin{array}{llll}1 & 1 & 1 & \ldots 1\end{array}\right]^{T}$ were chosen. Letting $\mathbf{x}=\Phi \mathbf{q}$, where $\Phi$ is the modal matrix and $\mathbf{q}$ are modal coordinates, equation (3) can be expressed as

$$
\Phi^{T} \mathbf{M} \Phi \ddot{\mathbf{q}}+\Phi^{T} \mathbf{K} \Phi \mathbf{q}=\mathbf{0},
$$

which is decoupled. The initial conditions for the modal coordinates were $\mathbf{q}(0)=\mathbf{0}$ and $\dot{\mathbf{q}}(0)=\Phi^{T} \mathbf{M} \dot{\mathbf{x}}(0)$. Solving equation (7) with the initial conditions provides the modal displacement vector $\mathbf{q}$, whence the full displacement vector is $\mathbf{x}=\Phi \mathbf{q}$. Retaining and sampling the sensed mass displacements $\mathbf{y}$, we obtained the reduced order ensemble matrix $\mathbf{Y}$. The modes were then estimated using equation (5).

Figure 1 compares the eight estimated reduced-mass weighted POMs (circles) with the associated full-order structural modes (dots connected by lines). The modes in Figure 1 are ordered according to the increasing modal frequency. From Figure 1 (a), (b), (c), (e), and (g), we see that the modes 1, 2, 3, 5 and 7 agree closely, while mode 4 in Figure 1 (d) is a rough approximation. With eight sensors, even if the higher modes match well, the full resolution modes are not accurately visualized from the POMs. The POVs were $1.06 \times 10^{4}, 82.8,25.4,6.65,1.80,1.26,0.282,0.107$, indicating the relative energy associated with the modes. The modal vectors ordered by decreasing POVs, or modal dominance, are modes 1, 3, 2, 5, 7 , 4, 8, and 6, corresponding to Figures 1 (a), (c), (b), (e), (g), (d), (h), and (f), respectively. The top five modes ordered by the POVs are the ones that were identified well. Mode 4 is low in this POV order, explaining why it is noticeably erroneous. Thus, with mere linear interpolation, the convergent results were quite good.

The effect of $N$ on the results was investigated by looking at the mean norm, $\delta$, of the difference (error) between unit modal vectors generated at $N=16000$ and the corresponding unit modal vectors generated for smaller values of $N$. The values of $\delta$ were small down to values around $N=2000$ (about two fundamental periods of data), and then increased markedly for lower values of $N$, suggesting that for $N>2000, N$ does not significanlty affect the estimation. For $N=250$, $500,750,1000,1250,1500,1750$, and 2000, the corresponding errors are $\delta=1.0206,0.0671,0.1496,0.5129,0.0680$, $0.0295,0.0657$, and 0.0437 . The vectors generated at $N=1000$, for which $\delta=0.5129$, are plotted with ' $\mathrm{x}$ ' symbols in Figure 1. For the five dominant modes, the $N=1000$ estimations overlap well with the $N=16000$ estimations. For the nethermost modes, the errors are large.

These results are for an undamped system. The interpretation of POD for modal analysis was derived for undamped systems, and tends to hold if the damping is light, e. g. in the range of physical experiments on beams and shells [18-20].

\section{Continuous Systems in One Dimension}

We consider vibration systems that are modeled by the partial differential equation $m(x) \ddot{u}+L_{o} u=0$, where $u(x, t)$ is a displacement (for example), $m(x)$ is a mass, $x$ is a spatial independent variable, and $L_{o}$ is a linear operator, with boundary 
conditions necessary for the order of $L_{o}$. The associated eigenvalue problem is

$$
\lambda m(x) \phi(x)=L_{o} \phi(x) .
$$

Measurements $y_{i}(t)=u\left(x_{i}, t\right)$ are made at locations $x_{i}, i=1, \ldots, n$. The measurements are sampled $N$ times at a uniform rate, as before, to build the correlation matrix $\mathbf{R}$. The task is then to construct an $n \times n$ mass matrix, $\mathbf{M}_{r}$, from the continuous mass distribution, for the mass weighted POD eigenvalue problem (5). We do this by using linear interpolation functions, analogous to the matrix $\mathbf{W}$ used in the discrete case.

\subsection{Example}

In this example we look at a one-dimensional continuous mass system with spatially varying mass, the exponential horn, or equivalently the "exponential rod", as its analytical solution is available.

We consider a second-order distributed parameter system equation of the form $m(x) \ddot{u}+\left(E A(x) u^{\prime}\right)^{\prime}=0$, with boundary conditions $u(0, t)=u(L, t)=0$, representing the axial vibration of a rod of length $L$, cross sectional area $A$ and elastic modulus $E$, where $u$ is the axial displacement and $x$ is the spatial independent variable. The dots and primes represent partial derivatives with respect to time and space, respectively. Given a mass per unit length $m(x)=m_{0} e^{\alpha x}$, and the product $E A(x)=E A_{0} e^{\alpha x}$, the equation of motion can be written as

$$
m_{0} \ddot{u}+E A_{0}\left(\alpha u^{\prime}+u^{\prime \prime}\right)=0 .
$$

The axial vibration of air in a tube (a horn) has a partial differential equation (PDE) of the same form. Separating variables as $u(x, t)=q(t) \phi(x)$ leads to

$$
\ddot{q}+\omega^{2} q=0,
$$

and

$$
\phi^{\prime \prime}+2 \zeta \Omega \phi^{\prime}+\Omega^{2} \phi=0 .
$$

with boundary conditions $\phi(0)=\phi(L)=0$, where $\alpha=2 \zeta \Omega$ and $\Omega^{2}=\frac{m_{0} \omega^{2}}{E A_{0}}$.

For the example of $m_{0}=E A_{0}=1$ we find that $\zeta<1$, and so this equation, in space, looks similar to a second-order free under-damped vibration equation in time. Equation (11) has the general solution $\phi=e^{-\zeta \Omega x}\left(A \cos \left(\Omega_{d} x\right)+B \sin \left(\Omega_{d} x\right)\right)$, where $\Omega_{d}=\Omega \sqrt{1-\zeta^{2}}$. Applying the boundary condition $\phi(0)=0$ we get $\phi=e^{-\zeta \Omega x} B \sin \left(\Omega_{d} x\right)$. Applying $\phi(L)=0$ suggests that $\Omega \sqrt{1-\zeta^{2}}=\frac{n \pi}{L}$. Inserting $\zeta=\frac{\alpha}{2 \Omega}$, and solving for $\Omega$ produces $\Omega_{n}^{2}=\left[(\alpha / 2)^{2}+(n \pi)^{2} / L^{2}\right]$, and thus

$$
\omega_{n}^{2}=\frac{E A_{0}}{m_{0}}\left[\left(\frac{\alpha}{2}\right)^{2}+\left(\frac{n \pi}{L}\right)^{2}\right]
$$

for $n=1,2, \ldots$ As such,

$$
\phi_{n}(x)=e^{-\alpha x / 2} \sin \left(\frac{n \pi x}{L}\right)
$$

are the eigen (modal) functions, and $q_{n}(t)=C \sin \omega_{n} t+D \cos \omega_{n} t$ are the modal coordinates.

We aim to simulate the free response of the horn, with displacements "measured" at eight equally spaced interior locations. We will then apply the discretized reduced mass-weighted POD to estimate the modal functions (13).

Applying the mass weighted POD requires the estimation of an $8 \times 8$ reduced-order mass matrix $\mathbf{M}_{r}$. The elements of the mass matrix $\mathbf{M}_{r}$ are determined as

$$
\hat{M}_{m n}=\hat{M}_{n m}=\int_{0}^{L} e^{\alpha x} \eta_{m}(x) \eta_{n}(x) d x,
$$


where $\eta_{m}(x)$ and $\eta_{n}(x)$ are tent-shaped interpolation functions chosen as $\eta_{m}(x)=\frac{1}{h}(x-(m-1) h)$ for $(m-1) h \leq x<$ $m h, \eta_{m}(x)=-\frac{1}{h}(x-(m+1) h)$ for $m h \leq x<(m+1) h$, and $\eta_{m}(x)=0$ otherwise. Here $m$ is an index for the measured displacement locations, ( $m=1,2, \ldots, 8$ in this example) and $h$ is the distance between sensors (uniform in this example).

We plug these functions into equation (14). For $m=n$, the elements of $\mathbf{M}_{r}$ are

$$
\begin{aligned}
\hat{M}_{m n} & =\int_{(m-1) h}^{m h} e^{\alpha x}\left[\frac{1}{h}(x-(m-1) h)\right]^{2} d x \\
& +\int_{m h}^{(m+1) h} e^{\alpha x}\left[\frac{1}{h}(x-(m+1) h)\right]^{2} d x,
\end{aligned}
$$

which, upon integration, produces

$$
\hat{M}_{m m}=\frac{-2\left(2 h \alpha+e^{-h \alpha}-e^{h \alpha}\right) e^{h m \alpha}}{h^{2} \alpha^{3}} .
$$

For $m=n-1$,

$$
\hat{M}_{m n}=-\int_{m h}^{(m+1) h} e^{\alpha x}\left[\frac{1}{h}(x-(m+1) h)\right]\left[\frac{1}{h}(x-(n-1) h)\right] d x
$$

which, upon integration, gives

$$
\hat{M}_{m n}=\frac{e^{h m \alpha}\left(2+h \alpha+e^{h \alpha}(-2+h \alpha)\right)}{h^{2} \alpha^{3}} .
$$

By symmetry, $\hat{M}_{m n}=\hat{M}_{n m}$. For $|n-m|>1, \hat{M}_{m n}=\hat{M}_{n m}=0$. $\mathbf{M}_{r}$.

For the case of eight equally spaced interior sensors, $h=L / 9$. Given a value of $\alpha$ and $L$, we can evaluate the mass matrix

For the simulation of the exponential horn, we converted the partial differential equation to a truncated set of modal coordinates, chose initial conditions in the modal coordinates, evaluated the modal coordinate response, and recombined them to approximate the horn response sampled at the values of $x=h, 2 h, \ldots, 8 h$.

There is a small detail to confront in this process, namely, that the system, as treated in equation (9) or (11), is non self adjoint. Working equation (9) or (11) into the form of equation (8), we have

$$
L_{o}=E A_{0}\left(\frac{d^{2}}{d x^{2}}+\alpha \frac{d}{d x}\right)
$$

as the linear operator. The adjoint operator $L_{o}^{*}$ is

$$
L_{o}^{*}=E A_{0}\left(\frac{d^{2}}{d x^{2}}-\alpha \frac{d}{d x}\right)
$$

Since $L_{o} \neq L_{o}^{*}$, the system is non self adjoint.

Modal analysis must be done by applying biorthogonality between modal functions of both the original and the adjoint eigenvalue problems [25]. To this end, we solve $\lambda m_{0} u=L_{o} u$ to obtain eigenfunctions $\phi_{n}(x)$, and $\lambda m_{0} v=L_{o}^{*} v$ to obtain adjoint eigenfunctions $\psi_{m}(x)$. By biorthogonality, $\left(\psi_{m}, m_{0} \phi_{n}\right)=\delta_{m n}$ and $\left(\psi_{m}, L_{o}^{*} \phi_{n}\right)=\lambda_{n}^{2} \delta_{m n}$. We let $u(x, t)=\sum_{k=1}^{\infty} q_{k}(t) \phi_{k}(x)$, insert into equation (9), multiply both sides by $\psi_{j}(x)$ and integrate. Biorthogonality properties decouple the system into infinitely many ordinary differential equations (ODEs) $\ddot{q}_{j}+\omega_{j} q=0$, of which we keep $N_{q}$ to form a truncated set. The solutions of these ODEs are $q_{j}(t)=A_{j} \sin \left(\omega_{j} t\right)+B_{j} \cos \left(\omega_{j} t\right)$.

We chose initial conditions such that $A_{j}=1 / j$ and $B_{j}=0$. The displacements $u(x, t)$ were obtained and then discretized by evaluating at the eight equally spaced values $x_{i}$, such that each discrete measured displacement is $y_{i}(t)=u\left(x_{i}, t\right)$. The discrete displacements $y(t)$, sampled at a constant rate, were then used to build the ensemble $\mathbf{Y}$, and hence $\mathbf{R}$. Then equation (5) was used to do the reduced mass weighted POD. 
We have performed this analysis on a numerically simulated dimensionless exponential horn with $m_{0}=A E_{0}=L=1$ and $\alpha=4$, sensed at 8 equally spaced locations, and sampled at a rate of $\Delta t=0.04$ for a total of $N=4800$ samples. We kept $N_{q}=10$ modal coordinates for the truncated modal analysis simulation. The modal frequencies of these ten modes varied from 2.5431 to 15.8348 . The simulation spanned about 78 fundamental periods, and the sampling was about ten times per highest-frequency-modal period. The initial velocity response specified above was used for the reduced mass-weighted POD. The mass-weighted POVs were 0.2459, 0.0577, 0.0234, 0.0115, 0.0061, 0.0035, 0.0021, and 0.0023. The order of modal dominance nearly matches the order of increasing frequencies because of the way the initial conditions were specified via the decreasing modal amplitudes $A_{j}$.

Figure 2 shows the mode shapes of the structural eigenvalue problem (solid lines) and the estimated mode shapes from the reduced mass-weighted POD (circles). From Figure 2 (a)-(f), we see very good agreement between the mode shapes as estimated by the mass-weighted POD and those of the structure. Although the mode shapes in Figures 2 (e) and (f) are in agreement, the spatial resolution is becoming difficult to fully capture with the limited number of sensors.

These results of the reduced mass-weighted POD based upon linear interpolation functions. The unweighted POD results are shown in Figure 2 with the ' $x$ ' symbols. Mass weighting is seen to be essential in producing good estimates of the modes for this example. Also, piecewise constant "interpolation" functions were used to form a diagonal lumped-mass matrix, and produced the results plotted with solid dots. For the first five modes, i.e. Figure 2 (a)-(e), the dots overlap the circles very closely. The estimates from linear interpolation functions remain somewhat close to the next two true modes of (f) and (g), while the estimates from the piecewise constant interpolations do not.

When the number of samples was reduced to 480, keeping the same sampling rate, the results for the first five modes were nearly the same, but the three highest modes showed significant differences. We also added random noise to each measured sample in ensemble $\mathbf{Y}$ by using the 'rand' function in Matlab. The random noise had zero mean and a peak-topeak range of $y_{m} 2^{-b}$, where $y_{m}$ is the maximum absolute value of the recorded displacement samples, and $b$ represents the reciprocal noise level in bits. As such, the expected mean squared value of the noise was $y_{m} 2^{-b} / 3$. With values of $b \geq 6$, there was no visible error in the mode shapes. With $b=4$, the noise effects were noticeable, but modes 1-3 were still very good. With $b=3$, modes 1 and 2 were good, and with $b=2$, all modes had noticeable error. Similar noise robustness was recorded in previous modal analyses by POD.

\section{Conclusions}

Mass-weighted POD can be performed for modal analysis for systems with light damping and multimodal free responses. A challenge is that the system may have more degrees of freedom than measurements, thereby making the mass and correlation matrices dimensionally incompatible. We presented a concept for reduced mass-weighted POD for modal analysis. A one-dimensional system can be reduced by an interpolated displacement strategy for effectively lumping the mass, so that POD modal analysis can be applied with a limited number of sensors. An approach was sketched for interpolation schemes for which the interpolated displacements (unmeasured) are linear combinations of the measured displacements.

Quality of the results will depend on the quality of knowledge of the mass distribution, as well as the quality of the interpolation scheme. We used linear interpolation and applied the reduced mass-weighted POD to a discrete example and a continuous example to show the feasibility. Even a piecewise constant interpolation was effective in the continuous example, while unweighted POD produced inaccurate results, suggesting that a simple effort to weight the mass properly can be significant.

We anticipate that this mass-reduction could be extended to situations where the mass matrix is obtained from an FEM formulation or some other design tool. Future work can address experiments, FEM interfaces, and also the extension of this idea to two-dimensional discrete and continuous systems such as plates and shells.

\section{Achnowledgements}

This material is based upon work supported by the National Science Foundation under Grant No. CMMI-0727838. Any opinions, findings, and conclusions or recommendations expressed in this material are those of the authors and do not necessarily reflect the views of the National Science Foundation.

\section{References}

[1] P. Avitabile, "Experimental modal analysis: A simple non-mathematical presentation," Sound and Vibration, vol. 35, pp. 20-31, January 2001.

[2] D. J. Inman, Engineering Vibration. Prentice Hall, Upper Saddle River., 2001.

[3] S. R. Ibrahim and E. C. Mikulcik, "A method for the direct identification of vibration parameters from the free response," Shock and Vibration Bulletin, vol. 47, no. 4, p. 183198, 1977. 
[4] H. Vold, J. Kundrat, G. Rocklin, and R. Russel, "A multi-input modal estimation algorithm for mini-computer," SAE Technical Papers Series, No 820194, vol. 91, pp. 815-821, 1982.

[5] J. N. Juang and R. Pappa, "An eigensystem realization algorithm for modal parameter identification and model reduction," Journal of Guidance, Control and Dynamics, vol. 8, no. 5, p. 620 627, 1985.

[6] D. L. Brown, R. J. Allemang, R. D. Zimmerman, and M. Mergeay, "Parameter estimation techniques for modal analysis," SAE Transactions, SAE Paper Number 790221, vol. 88, pp. 828-846, 1979.

[7] P. Overshee and B. D. Moor, Subspace Idenification for Linear Systems, Theory Implementation, Application. Kluwer Academic Publishers, 1996.

[8] H. Vold, "Orthogonal polynomials in the polyreference method," in Proceedings of the International Seminar on Modal Analysis, Katholieke University of Leuven, Belgium, 1986.

[9] M. Richardson and D. L. Formenti, "Parameter estimation from frequency response measurements using rational fraction polynomials," in Proceedings of the International Modal Analysis Conference, pp. 167-182, 1982.

[10] C. Y. Shih, Y. G. Tsuei, R. J. Allemang, and D. L. Brown, "Complex mode indication function and its application to spatial domain parameter estimation," Mechanical System and Signal Processing, vol. 2, pp. 367-377, 1988.

[11] R. Brincker, L. Zhang, and P. Andersen, "Modal identification of output-only systems using frequency domain decomposition," Smart Materials And Structures, vol. 10, pp. 441-445, 2001.

[12] W. Zhou and D. Chelidze, "Generalized eigenvalue decomposition in time domain modal parameter identification," Journal of Vibration and Acoustics, vol. 130, no. 1, p. 011001, 2008.

[13] W. Liu, W.-C. Gao, and Y. Sun, "Application of modal identication methods to spatial structure using field measurement data," Journal of Vibration and Acoustics, vol. 131, no. 3, p. 034503, 2009.

[14] B. Feeny and R. Kappagantu, "On the physical interpretation of proper orthogonal modes in vibrations," Journal of Sound and Vibration, vol. 211, no. 4, pp. 607-616, 1998.

[15] B. F. Feeny, "On the proper orthogonal modes and normal modes of continuous vibration systems," Journal of Vibration and Acoustics, vol. 124, no. 1, pp. 157-160, 2002.

[16] G. Kerschen and J. C. Golinval, "Physical interpretation of the proper orthogonal modes using the singular value decomposition," Journal of Sound and Vibration, vol. 249, no. 5, pp. 849-865, 2002.

[17] B. F. Feeny and Y. Liang, "Interpreting proper orthogonal modes in randomly excited vibration systems," Journal of Sound and Vibration, vol. 265, no. 5, pp. 953-966, 2003.

[18] S. Han and B. F. Feeny, "Application of proper orthogonal decomposition to structural vibration analysis," Mechanical Systems and Signal Processing, vol. 17, no. 5, pp. 989-1001, 2003.

[19] M. S. Riaz and B. F. Feeny, "Proper orthogonal decomposition of a beam sensed with strain gages," Journal of Vibration and Acoustics, vol. 125, no. 1, pp. 129-131, 2003.

[20] U. Iemma, L. Morino, and M. Diez, "Digital holography and Karhunen-Loève decomposition for the modal analysis of two-dimensional vibrating structures," Journal of Sound and Vibration, vol. 291, pp. 107-131, 2006.

[21] D. Chelidze and W. Zhou, "Smooth orthogonal decomposition-based vibration mode identification," Journal of Sound and Vibration, vol. 292, pp. 461-473, 2006.

[22] B. F. Feeny and U. Farooq, "A nonsymmetric state-variable decomposition for modal analysis," Journal of Sound and Vibration, vol. 310, no. 4-5, pp. 792-800, 2008.

[23] A. Przekop, X. Guo, and S. A. Rizzi, "Alternative modal basis selection procedures for nonlinear random response simulation," in Proceedings of the 10th International RASD Conference, (Southampton), July 12-14 2010.

[24] G. M. Forsythe, M. A. Malcolm, and C. B. Moler, Computer Methods for Mathematical Computations. Englewood Cliffs: Prentice-Hall Series in Automatic Computation, 1977.

[25] L. Meirovitch, Principles and Techniques in Vibrations. Prentice Hall, 1997.

[26] B. I. Epureanu and E. H. Dowell, "Localized basis function method for computing limit cycle oscillations," Nonlinear Dynamics, vol. 31, pp. 151-166, 2003. 


\section{Figure Captions}

Figure 1. Estimated mode shapes from the reduced mass-weighted POD obtained from 16 fundamental periods (denoted by circles) and one fundamental period (' $x$ ' symbols) of data, in comparison with the 24-mass structural modal vectors (dots connected by lines). (a) shows the mode shape associated with the lowest modal frequency, and (b)-(h) show the modes shapes associated with increasing modal frequencies.

Figure 2. Estimated mode shapes of the exponential rod from the reduced mass-weighted POD obtained using linear interpolation functions (denoted by circles), piecewise constant interpolation functions (dots), and unweighted POD (' $\mathrm{x}$ ' symbols), in comparison with structural modal functions (denoted by lines). (a) shows the mode shape associated with the lowest modal frequency, and (b)-(h) show the modes shapes associated with increasing modal frequencies. 

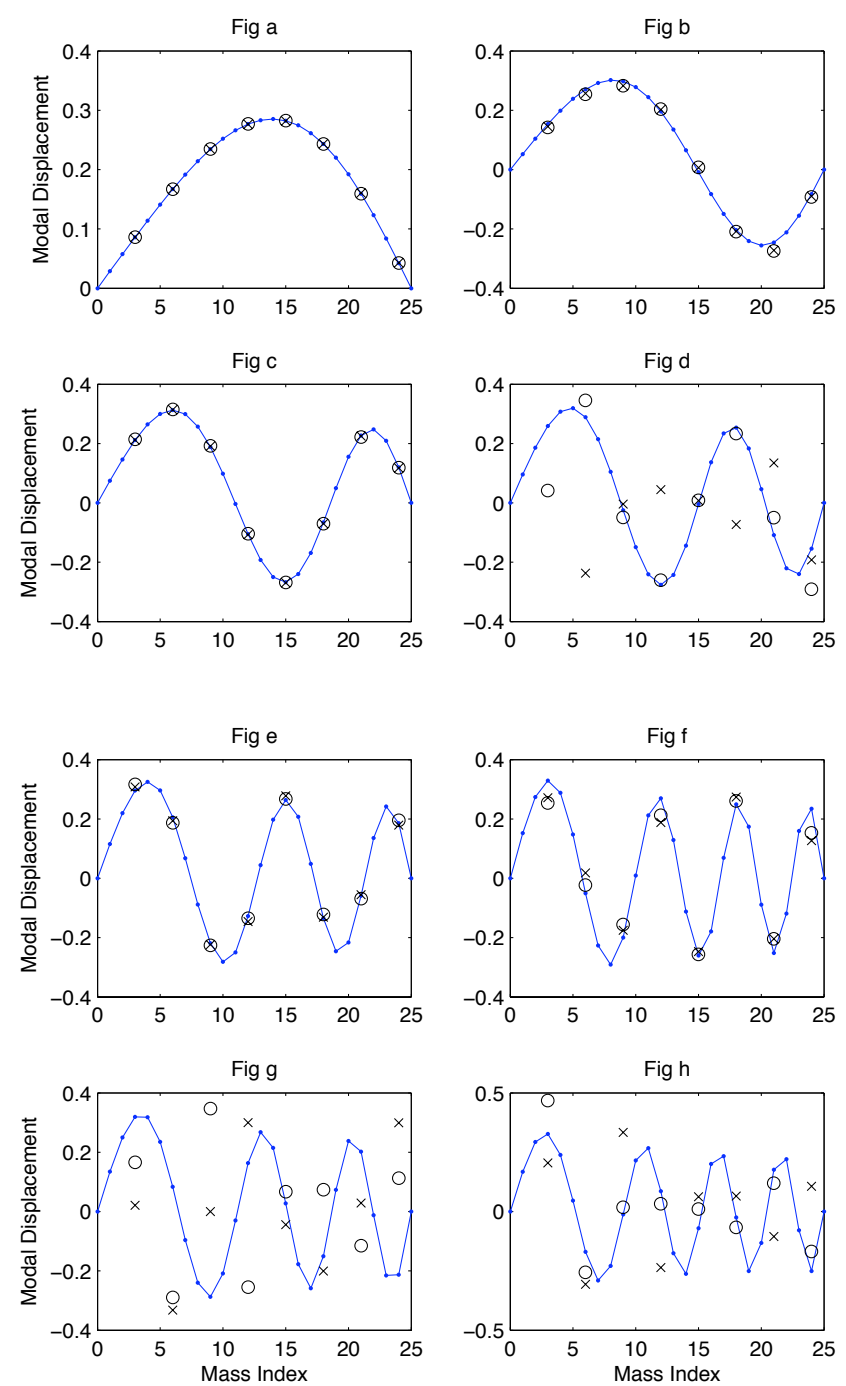

Fig. 1. Estimated mode shapes from the reduced mass-weighted POD obtained from 16 fundamental periods (denoted by circles) and one fundamental period (' $x$ ' symbols) of data, in comparison with the 24-mass structural modal vectors (dots connected by lines). (a) shows the mode shape associated with the lowest modal frequency, and (b)-(h) show the modes shapes associated with increasing modal frequencies.

Files Figure1(a)-(d).pdf and Figure1(e)-(h).pdf

VIB-09-1192, Yadalam and Feeny, page 9 

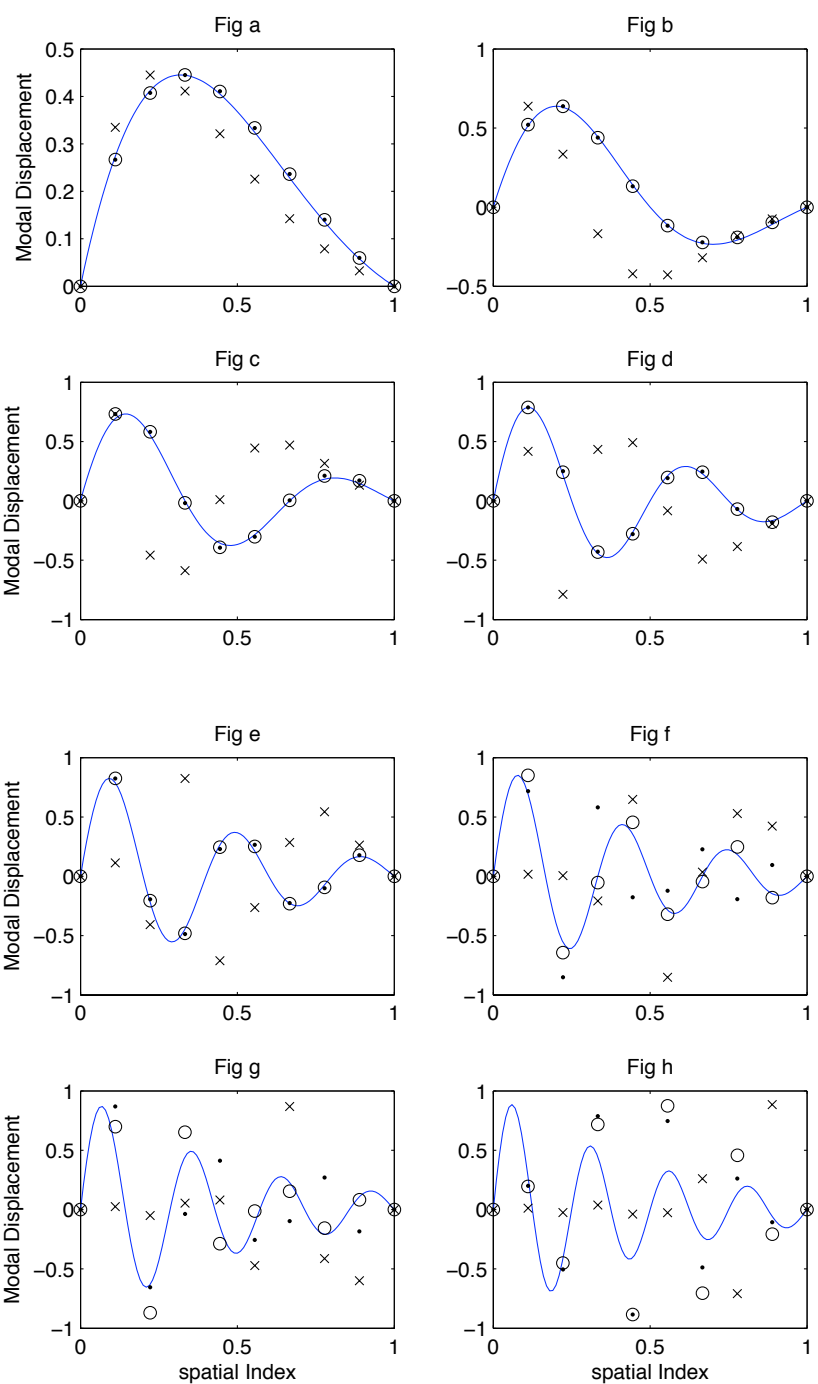

Fig. 2. Estimated mode shapes of the exponential rod from the reduced mass-weighted POD obtained using linear interpolation functions (denoted by circles), piecewise constant interpolation functions (dots), and unweighted POD (' $x$ ' symbols), in comparison with structural modal functions (denoted by lines). (a) shows the mode shape associated with the lowest modal frequency, and (b)-(h) show the modes shapes associated with increasing modal frequencies.

Files Figure2(a)-(d).pdf and Figure2(e)-(h).pdf

VIB-09-1192, Yadalam and Feeny, page 10 